\title{
SOME ASPECTS AND EXPERIMENTS ON THE DENSITY CURRENT
}

(ON THE INTERNAL WAVES)

\author{
By Hiroyoshi Shi-igai*, C. E. Member
}

\author{
Nomenclature \\ $h:$ depth of layers \\ $u$ : velocity component of $x$-direction \\ $q:$ discharge per unit width \\ $\Delta \rho:$ difference of density between two layers \\ $c$ : wave velocity of propagation \\ $F_{i}:$ internal Froude number \\ $\stackrel{\nabla}{\equiv}$ : surface of water \\ $H$ : total depth of layers \\ $w$ : velocity component of $y$-direction \\ $\rho:$ density \\ $\varepsilon=\frac{\Delta \rho}{\rho}$ \\ $F:$ Froude number \\ $g:$ gravity acceleration \\ $\nabla:$ interface \\ * Suffix 1 means the value with respect to the \\ upper layer and suffix 2 with respect to lower, \\ or under-layer.
}

\section{Chapter 1. Introduction}

\subsection{On the Density Current}

When we talk adout so-called density current, we, the author believes, cannot be so sure of having definite conceptions on it. Sometimes the phenomena that civil engineers call density current have absolutely different names in the field of meteorology or hydro-dynamics. Even in the field of hydraulics we may treat some of the problems on sedimentation and those on air entrained flow as the density current.

Besides them there are many unsolved or not completely solved problems on the density current in civil engineering; they are, for example, the selective withdrawal from the multiple layered system, the characteristics of the internal waves, intrusion of pure water into salt water, the behaviour of the thermal density current, etc.

One of the reasons why these problems are difficult to solve is lack of numbers of applicable equations. If the motion of fluid is considered in three dimensions, we can immediately

\footnotetext{
* Graduate School, Civil Engineering, University of Tokyo.
}

use four equations tosolve it, (one of which is equation of continuity,) the others of which are equations of motion. On the other hand five unknowns are included in the problem; they are three cemponents of velocity, pressure and density. If the fluid is compressible, we can use equation of state as the fifth equation. In the case of incompressible fluid, however, the suitable fifth equation has not been foundout. We may of course adopt the equation of conservation of concentration or some particles in liquid. (Here concentration may be of salt, silt, etc.) This means a new variable comes into sight again and situation is never improved. One would say that as the sixth equation the relation between concentration and density is applicable. This relation, however, has too simple form usually and it sometimes happens that the equation of continuity and that of conservation of concentration have just the same meaning with each other.

To solve the problems, therefore, one would often assume some condition at the interface It would be of importance that we must have some definite conceptions on it. The author tries to form some definitions on the density current to establish the rigid conceptions.

\subsection{Some Definitions on the Density Current}

i) Definition of the density current

Density current is a current of incompressible fluid* affected by its density gradient or distribution.

ii) Definition of the perfect density current.

Perfect density current is a density current caused by several kinds of perfect liquid with difflerent densities which cannot be mixed with each other.

iii) Definition of the unordinary density current.

\footnotetext{
* Here the author will not refer to the density current of compressible fluid. There needs some preparations for it.
} 
Unordinary density current is a density current caused by several kinds of viscous liquid which cannot be mixed with each other.

iv) Definition of the ordinary density current. Ordinary density current is a density current caused by several kinds of viscous liquid which can be mixed with each other.

\subsection{Some Remarks}

Perfect density current cannot be really seen as well as perfect liquid. Yet, to assume some motion as the perfect density current is one of the useful methods in solving problems. To solve the internal or interfacial wave problem you will find this assumption is useful.

Unordinary density current can be seen; that is, for example, the case of two-layered motion of oil and water, or water and air which is treated as incompressible. We must keep it in mind the affection of viscosity or shearing stress at the interface. Here we need not think of diffusion through the interface. We can treat the motion of salt water and pure water as an unordinary density current where turbulent diffusion is very small.

The author will show some aspects and experiments on the density current in the following chapters.

\section{Chapter 2. Internal Solitary Wave}

\subsection{On the Internal waves with small amplitude}

On the internal waves which have an infinitesimal amplitude there are many works such as

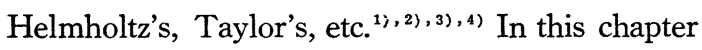
the author gives some considerations and experimental results on the internal solitary wave.

At the interface of two-layered system the velocity of propagation of the internal wave with small amplitude is given in such a wellknown form.

$$
c=\sqrt{\frac{g}{m} \frac{\Delta \rho}{\rho_{2} \operatorname{coth} m h_{2}+\rho_{1} \operatorname{coth} m h_{1}}} \cdots \cdots \cdots(1)
$$

where $m=2 \pi / \lambda$, and $\lambda$ is the wave length.

In eq. (1) $\operatorname{cothm} h_{2}$ tends to unity as $h_{2}$ diverges. Furthermore, where $\lambda$ is very large compared to $2 \pi$, we obtain

$$
\lim _{\lambda \rightarrow \infty} \frac{2 \pi}{\lambda} \operatorname{coth} \frac{2 \pi}{\lambda} h=\frac{1}{h}
$$

Consequently we can adopt two kinds of wave velocities in the limit case as following.

$$
\left.\begin{array}{cc}
c_{1}=\sqrt{g \frac{\Delta \rho h_{1} h_{2}}{\rho_{2} h_{1}+\rho_{1} h_{2}}} & (\lambda \rightarrow \infty) \\
c_{2}=\sqrt{g \frac{\Delta \rho h_{1}}{\rho_{1}}} & \left(\begin{array}{l}
\lambda \rightarrow \infty \\
h_{2} \rightarrow \infty
\end{array}\right)
\end{array}\right\}
$$

Equa-tion (2) corresponds to the equation of velocity of propagation by the ordinary long waves but that $g$ is changed into $\frac{\Delta \rho}{\rho} \mathrm{g}$, and it is interesting that by the internal solitary wave the underlayer depth $h_{2}$, where it is large compared to that of upper-layer, affects on its behaviour not so much as the depth of upperlayer $h_{1}$ and density difference $\Delta \rho$ do. Furthermore, the ordiary solitary wave never occurs whenever the water depth is very large compared to its amplitude. But in case of the internal solitary wave it can occur where either of two kinds of depth is shallow.

\subsection{Analysis}

Fig. 1 shows the co-ordinate system of the two-layered flow. Let the water surface be $x$ axis and take $y$-axis downward. At first we investigate the phenomena where the amplitude of the internal solitary wave $2 \eta$ is very small compared to the depths $h_{1}$ and $h_{2}$. This problem will be treated as that of two dimensions and

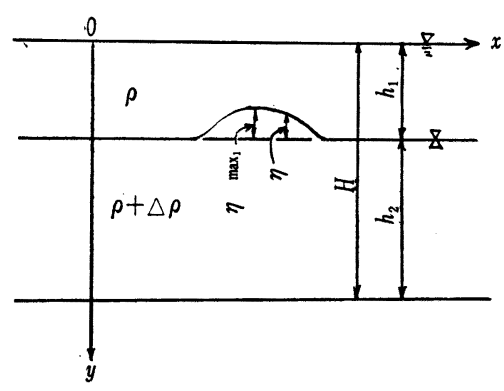

Fig. 1 Co-ordinate of two-Layer system.

of the perfect density current. The author will follow the well-known method of Boussinesq which was used $\mathrm{n}$ order to analyze the ordinary solitary wave.

At the interface pressure $p$ has to be continuous, so we may write from this boundary condition

$$
\begin{aligned}
& (\rho+\Delta \rho)\left[-\frac{\partial \phi}{\partial t}+g\left(h_{1}-\eta\right)+\frac{1}{2}\left(u^{2}+w^{2}\right)\right] \\
& =\rho g\left(h_{1}-\eta\right)
\end{aligned}
$$

where $\phi$ means the velocity potential.

As an internal wave suddenly occurs at the interface, the vertical velocity component $w$ is 
shown as follows.

$$
w=-\frac{\partial \eta}{\partial t}-\frac{\partial \eta}{\partial x} v
$$

At the bottom as the boundary conditions we may write

$$
\frac{\partial \phi}{\partial y}=0, \quad \nabla^{2} \phi=0
$$

Developping the velocity potential $\phi$ by powers of $y^{n}$, we obtain

$$
\phi=\sum_{n=0}^{\infty} \phi_{n} y^{n}, \quad \phi_{n}=\phi_{n}(x, t)
$$

We must define $\phi^{n}$ so as to $\nabla^{2} \phi=0$, and we get

$$
\phi=\phi_{0}-\frac{y^{2}}{2 !} \frac{\partial^{2} \phi_{0}}{\partial x^{2}}+\frac{y^{4}}{4 !} \frac{\partial^{4} \phi_{0}}{\partial x^{4}}-\frac{y^{6}}{6 !} \frac{\partial^{6} \phi_{0}}{\partial x^{6}}+\cdots \cdots
$$

using the condition of infinitesimal wave height, or $\frac{\Delta \rho}{\rho} g h=g^{\prime} h \gg u^{2}, w^{2}$, we may write

$$
-\frac{\partial \phi}{\partial t}-g \frac{\Delta \rho}{\rho+\Delta \rho}\left(h_{1}-\eta\right)=-\frac{\partial \phi}{\partial t}-g h^{\prime}
$$

where $h^{\prime}=\frac{\Delta \rho}{\rho+\Delta \rho}\left(h_{1}-\eta\right)$. Here we obtain

$$
\left.\begin{array}{c}
-\frac{\partial \phi}{\partial t}-g h^{\prime}=0 \\
\frac{\partial \phi}{\partial y}-\frac{\partial \eta}{\partial t}=0
\end{array}\right\}
$$

Considering $\phi_{0} \gg \frac{\partial^{2} \phi_{0}}{\partial x^{2}} \gg \frac{\partial^{4} \phi_{0}}{\partial x^{4}}$ as the first approximation, eq. (10) is obtained by substitution of eq. (9) into eq. (8).

$$
\left.\begin{array}{l}
-\frac{\partial \phi_{0}}{\partial t}-g h^{\prime}=0 \\
-y \frac{\partial^{2} \phi_{0}}{\partial x^{2}}-\frac{\partial \eta}{\partial t}=0
\end{array}\right\}
$$

Considering the facts that as $\eta$ is very small compared to $h_{1}, y \fallingdotseq h_{1}$ and that $g \frac{\partial^{2} h^{\prime}}{\partial x^{2}}=-\frac{\Delta \rho}{\rho+\Delta \rho}$ $g \frac{\partial^{2} \eta}{\partial x^{2}}$, we obtain

$$
\frac{\partial^{2} \eta}{\partial t^{2}}=g^{\prime} h_{1} \frac{\partial^{2} \eta}{\partial x^{2}}
$$

And this is the equation of wave propagation by the velocity of propagation $c=\sqrt{g^{\prime} h_{1}}$ where $g^{\prime}=\frac{\Delta \rho}{\rho+\Delta \rho} g$.

Next we consider the case in which the amplitude is comparably large.

Let $u=-\sqrt{\frac{g^{\prime}}{h_{1}}} \cdot \eta$ and substituting it into eq. (3), it may be written in the form

$$
-\frac{\partial \phi}{\partial t}+g h^{\prime}+\frac{1}{2} \frac{g^{\prime}}{h_{1}} \eta^{2}=0
$$

On the other hand substituting eq. (11) into eq. (4), the following relation is obtained.

$$
w=-\frac{\partial \phi}{\partial y}=-\frac{\partial \eta}{\partial t}+\sqrt{\frac{g}{h_{1}}} \eta \frac{\partial \eta}{\partial x}
$$

Thus we get a pair of fundamental equations as follows.

$$
\begin{gathered}
-\frac{\partial \phi}{\partial t}+g h^{\prime}+\frac{1}{2} \frac{g^{\prime}}{h_{1}} \eta^{2}=0 \ldots \ldots \\
\frac{\partial \phi}{\partial y}-\frac{\partial \eta}{\partial t}+\sqrt{\frac{g^{\prime}}{h_{1}}} \eta \frac{\partial \eta}{\partial x}=0
\end{gathered}
$$

After developping these equations with respect to $\phi$ and $\frac{\partial \phi}{\partial y}$, we get as the second order approximation

$$
\begin{aligned}
& \phi=\phi_{0}-\frac{h_{1}^{2}}{2} \frac{\partial^{2} \phi_{0}}{\partial x^{2}} \\
& \frac{\partial \phi}{\partial y}=-y \frac{\partial^{2} \phi_{0}}{\partial x^{2}}+\frac{y^{3}}{6} \frac{\partial^{4} \phi_{0}}{\partial x^{4}}+\cdots \cdots
\end{aligned}
$$

Partially differenciating eq. (16) with respect to $t$ and using eq. (10) and eq. (11), eq. (14) reads

$$
g h^{\prime}-\frac{\partial \phi_{0}}{\partial t}+\frac{h_{1}{ }^{2}}{2} \frac{\partial^{3} \phi_{0}}{\partial^{2} x \partial t}+\frac{1}{2} \frac{g^{\prime}}{h_{1}} \eta^{2}=0 \cdots
$$

Furthermore, considerihg that $\frac{\partial^{3} \phi_{0}}{\partial^{2} x \partial t}=g \frac{\partial^{2} h}{\partial x^{2}}$ $=-g^{\prime} \frac{\partial^{2} \eta}{\partial x^{2}}$, we may write

$$
g h^{\prime}-\frac{\partial \phi_{0}}{\partial t}-\frac{1}{2} g^{\prime}\left(\frac{\eta^{2}}{h_{1}}-h_{1} \frac{\partial^{2} \eta}{\partial x^{2}}\right)=0 \cdots
$$

From eq. (18) and eq. (15), we get a new equation by the virtue of the fact that $\frac{\partial \phi_{0}}{\partial x} \risingdotseq \sqrt{\frac{g^{\prime}}{h_{1}}} \eta$ as follows

$$
-\frac{\partial \eta}{\partial t}+H \frac{\partial^{2} \phi_{0}}{\partial x^{2}}+\sqrt{g^{\prime} h_{1}} \frac{\partial}{\partial x}\left(-\frac{\eta^{2}}{h_{1}}-\frac{h_{1}{ }^{2}}{6} \frac{\partial^{2} \eta}{\partial x^{2}}\right)
$$

$$
=0 \text {. }
$$

Eliminate $\phi_{o}$ from eq. (19) and (20), and use the approximation that $\partial t \fallingdotseq-\frac{\partial x}{\sqrt{g^{\prime} h_{1}}}$, and we obtain

$$
\begin{aligned}
& -\frac{\partial^{2} \eta}{\partial t^{2}}=g h^{\prime} \frac{\partial^{2}}{\partial x^{2}}\left(\frac{3}{2} \frac{\eta^{2}}{h_{1}}-\frac{1}{3} \frac{\partial^{2} \eta}{\partial x^{2}} h_{1}{ }^{2}\right) \\
& -g^{\prime} h_{1} \frac{\partial^{2} \eta}{\partial x^{2}}
\end{aligned}
$$

Let us use the definition of the velocity of propagation $c_{1}$ of the internal solitary wave, which suffices for the following equation.

$$
+\frac{\partial \eta}{\partial t}+\frac{\partial}{\partial x}\left(\eta c_{1}\right)=0
$$

From this eq. (21) will be

$$
\begin{aligned}
& -\frac{\partial}{\partial t}\left(\eta c_{1}\right)-g^{\prime} h_{1} \frac{\partial \eta}{\partial x} \\
& \quad+g^{\prime} h_{1} \frac{\partial}{\partial x}\left(\frac{3}{2} \frac{\eta^{2}}{h_{1}}-\frac{h_{1}{ }^{2}}{3} \frac{\partial^{2} \eta}{\partial x^{2}}\right)=0
\end{aligned}
$$

Next as the second order approximation let $c_{1}=\sqrt{g^{\prime} h_{1}}(1+\varepsilon)\left(\right.$ consider $\frac{\partial \eta}{\partial t}=\frac{\partial}{\partial x}\left(\eta c_{1}\right)$ and $\left.\partial t=\frac{\partial x}{\sqrt{g^{\prime} h_{1}}}\right)$ where $\varepsilon$ is a very small constant, and eq. (23) will read 


$$
\begin{aligned}
& \sqrt{g^{\prime} h_{1}}\left(-1+\frac{\eta}{2 h_{1}}\right)\left(\frac{\partial\left(\eta c_{1}\right)}{\partial x}+\varepsilon \sqrt{g^{\prime} h_{1}} \frac{\partial \eta}{\partial x}\right) \\
& -g^{\prime} h_{1} \frac{\partial \eta}{\partial x}+g^{\prime} h_{1} \frac{\partial}{\partial x}\left(\frac{3 \eta^{2}}{2 h_{1}}-\frac{1}{3} h_{1}{ }^{2} \frac{\partial^{2} \eta}{\partial x^{2}}\right) \\
& =0 \text {. }
\end{aligned}
$$

If we take this intarnal solitary wave as of parmanent type, $c_{1}=c$, where $c$ is the ordinary velcoity of propagation, so that we have

$$
2 h_{1} \frac{\partial^{2} \eta}{\partial X^{2}}=6\left(\frac{c^{2}}{g^{\prime} h_{1}}-1\right) \cdot \frac{\eta}{h_{1}}+9 \frac{\eta^{2}}{h_{1}{ }^{2}}+\varepsilon \cdot \cdot
$$

where $X=x-c t$, and $C$ is an integral constant which should be zero from the boundary conditions.

Integrating eq. (25) multiplied $\eta_{x}$ on its both sides, we get

$$
\left(\frac{\partial \eta}{\partial X}\right)^{2}=\frac{3 \eta^{2}}{h_{1}^{3}}\left\{\left(\frac{c^{2}}{g^{\prime} h_{1}}-1\right) h_{1}+\eta\right\}
$$

where at $X=\infty \eta$ and $\eta_{x}$ are equal to zero.

Integrating eq. (26) again and finally we get an equation which should state the form of the internal solitary wave.

$$
\eta=-\eta_{\max } \operatorname{sech}^{2} \sqrt{\frac{3}{4} \frac{\eta_{\max }}{h_{1}}} \frac{X}{h_{1}}
$$

This shows that the internal solitary wave may occur under the condition of $h_{1}$ being shallow, even if the total depth $h$ is very large. We can have a negative solitary wave also by putting $\eta=-\eta^{\prime}$.

Fig. 2 shows the relation between calculated

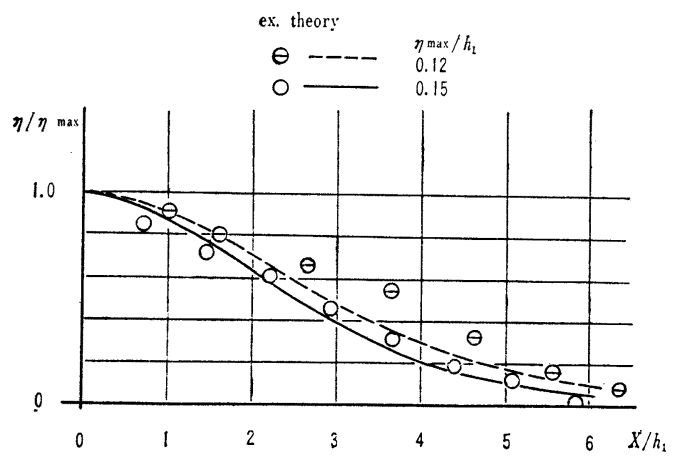

Fig. 2 Comparison of results of experiment and theory for the internal solitary wave.

value and experimental date. Considering the fact that the measurement of the internal solitary wave is rather difficult, Fig. 2 may show rather good coincidence between theory and experiment.

\subsection{Method to Measure the Internal Waves}

It seems one of the difficult problems to

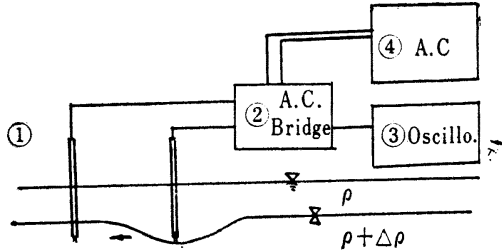

Fig. 3 Schematic view showing the method to measure the internal solitary wave.

measure the wave length and wave height of

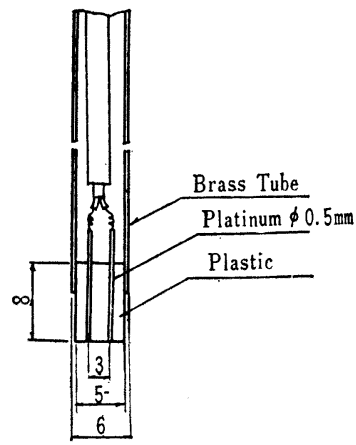
the internal waves. Fig. 3 shows the method to measure them. 1 is the electrode shown in Fig. 4 in details. 2 is an A.C bridge and 3 is the oscillograph which records electric signals from electrodes, which mean the change of electro conductivity of liquid. (Salt water was

Fig. 4 Electrode or probe. used as the lower liquid.)

To measure the amplitude of the internal wave by such probes we need several assumptions as follows;

1. The amplitude is to be smaller than at least one-fifth of the depth of the upper layer.

2. Density gradient near the interface is almost linear.

3. To fulfill the conditions of the internal solitary wave, the amplitude should be not so small compared to the depth of the upper layer. From those assumptions the following method was used.

At first, vertical density distribution is measured by probes. Fig. 5 shows one of the density distributions measured in the experiment. We can recognize from this the assumstion 2 is nearly established. Next, probes are set at a certain depth, which corresponds to the upper one-fourth of density changing region.

The channel used for this experiment is $2 \mathrm{~m}$ in length, $0.1 \mathrm{~m}$ in width and $0.6 \mathrm{~m}$ in depth. The internal solitary wave is easily generated by sinking something heavy in the small pool of $0.6 \mathrm{~m} \times 0.6 \mathrm{~m}$ attached to one end of the channel. As the internal wave thus generated 


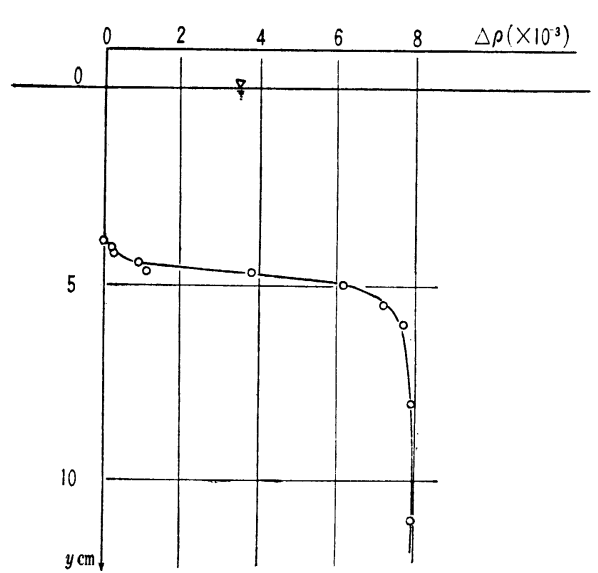

Fig. 5 An example of the density distribution near the interface.

propagates into the channel, conductivity changes according to the wave height, which the oscillograph records through probes. Using the calibration curve we can get a wave profile from the recorded data. These are shown in Fig. 6 schematically.

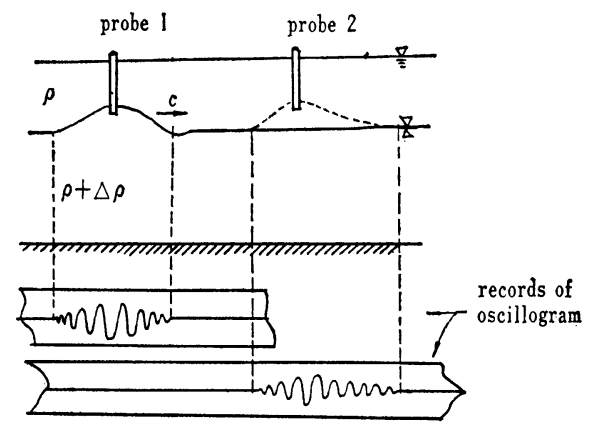

Fig. 6 An internal wave and its oscillogram.

\section{Chapter 3. On the Internal Jump}

\subsection{Theoretical Consideration}

There is another important and interesting problem on the internal wave; this is the internal jump in two-layer system. On this problem there is an excellent work of Chia-Shun, $\mathrm{Yih}^{5}$. According to his theory the author will research its behaviour, energy change and show some applications.

The motion is treated in two dimensions and in rectangular channel. Fig. 7 shows the schematic view of an internal jump in twolayer system. Here we take it as a perfect density current.

By the internal jump it is reasonable to assume for momentum to be preserved. If mo-

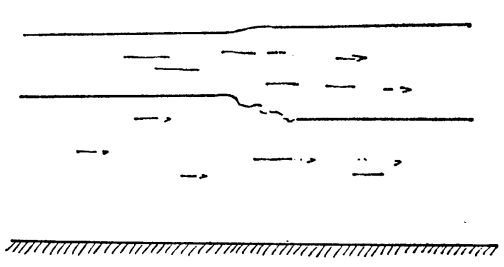

Fig. 1 Internal jump in two-layer system.

mentum difference between two sections I and II is equal to difference of the hydrostatic pressure, we obtain two momentum equations following.

$$
\left.\begin{array}{c}
\rho_{1} u_{1}{ }^{2} h_{1}-\rho_{1} u^{\prime 2} h_{1}{ }^{\prime}=\frac{1}{2} \rho_{1} g h_{1}{ }^{2}-\frac{1}{2} \rho_{1} g h_{1}{ }^{2} \\
\rho_{2} u_{2}{ }^{2} h_{2}-\rho_{2} u_{2}{ }^{2} h_{2}{ }^{\prime}=\frac{1}{2} \rho_{2} g h_{2}{ }^{2}+\rho_{1} g\left(h_{1} h_{2}\right. \\
\left.-\left(\frac{1}{2} \rho_{2} g\right) h_{2}{ }^{2}+\rho_{1} g h_{1}{ }^{\prime} h_{2}{ }^{\prime}\right)
\end{array}\right\}
$$

If there does not exist any mixing through the interface, we can write the equation of continuity as follows.

$$
\left.\begin{array}{l}
q_{1}=u_{1} h_{1}=u_{1}{ }^{\prime} h_{1}{ }^{\prime} \\
q_{2}=u_{2} h_{2}=u_{2}{ }^{\prime} h_{2}{ }^{\prime}
\end{array}\right\}
$$

Substituting equation (29) into eq. (28), we get

$$
\begin{gathered}
2 a_{2}\left(h_{2}-h_{2}{ }^{\prime}\right)=h_{2} h_{2}{ }^{\prime}\left(h_{2}+h_{2}{ }^{\prime}\right) \\
\cdot\left[\gamma\left(h_{1}-h_{1}{ }^{\prime}\right)+\left(h_{2}-h_{2}{ }^{\prime}\right)\right] \\
2 a_{1}\left(h_{1}-h_{1}{ }^{\prime}\right)=h_{1} h_{1}{ }^{\prime}\left(h_{1}+h^{\prime}{ }^{\prime}\right) \\
\cdot\left[\left(h_{1}-h_{1}{ }^{\prime}\right)+\left(h_{2}-h_{2}{ }^{\prime}\right)\right]
\end{gathered}
$$

where $a_{1}=\frac{q_{1}{ }^{2}}{g}, a_{2}=\frac{q_{2}{ }^{2}}{g}$ and $r=\frac{\rho_{1}}{\rho_{2}}$.

If we consider a case where only the upper layer moves and the lower ceases, $u_{2}=0$ in equation (30), so that we obtain

$$
\begin{aligned}
& -\left(h_{2}-h_{2}{ }^{\prime}\right)=r\left(h_{1}-h_{1}{ }^{\prime}\right) \ldots \ldots \ldots \ldots \ldots \ldots \\
& 2 a_{1}\left(h_{2}-h_{2}{ }^{\prime}\right)=h_{1} h_{1}{ }^{\prime}\left(h_{1}-h_{1}{ }^{\prime}\left(h_{1}-h_{1}{ }^{\prime}\right)\right) \varepsilon \cdots
\end{aligned}
$$

Eq. (33) may be written in another form by some non-dimensional variables.

$$
\left.\begin{array}{l}
2 F_{i}{ }^{2}(1-\xi)=\xi(1+\xi)(1-\xi) \\
F_{i}{ }^{2}=\frac{a_{1}}{\varepsilon h_{1}{ }^{3}} \\
\xi=\frac{h_{1}{ }^{\prime}}{h_{1}}
\end{array}\right\} \ldots \ldots \ldots
$$

Eq. (34) is a cubic equation in regard to $\xi$, and its there roots can easily be found; they are

$$
\begin{aligned}
& \xi_{10}=1 \\
& \xi_{20}=\frac{1}{2}\left(-1+\sqrt{1+8 F_{i}{ }^{2}}\right) \\
& \xi_{30}=\frac{1}{2}\left(-1-\sqrt{1+8 F_{i}{ }^{2}}\right)
\end{aligned}
$$

Since $\xi_{30}<0$, it is meaningless from physical point of view; $\xi_{10}$ is considered as an extra 
case, so that we should examine $\xi_{20}$.

(One can easily see that if we substitute the ordinary Froude number $F$ in place of $F_{i}$ into eq. (36), we get the ordinary wellknown equation of hydraulic jumps.)

From eq. (32) total depth change $\Delta H$ is

$$
\Delta H \equiv H-H^{\prime}=\varepsilon\left(h_{1}-h_{1}{ }^{\prime}\right) \cdot
$$

\subsection{Energy Change of the Internal Jump}

In this paragraph the author will calculate the energy change of the internal jump as is shown in Fig. 8 to determin if an internal jump can occur or not. Equations of specific energy of two sections $I$ and II are

$$
\left.\begin{array}{l}
E_{1}=\frac{u_{1}{ }^{2}}{2 g}+\frac{\rho_{2}}{\rho_{1}} \frac{u_{2}^{2}}{2 g}+h_{1}+\frac{\rho_{2}}{\rho_{1}} h_{2} \\
E_{2}=\frac{u_{1}{ }^{\prime}}{2 g}+\frac{\rho_{2}}{\rho_{1}} \frac{u_{2}{ }^{2}}{2 g}+h_{1}{ }^{\prime}+\frac{\rho_{2}}{\rho_{1}} h_{2}{ }^{\prime}
\end{array}\right\}
$$

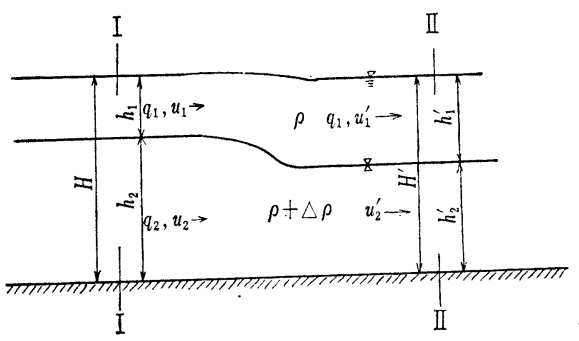

Fig. 8 Normal internal jump.

The energy change between two sections $\Delta E$ is

$$
\begin{aligned}
\Delta E= & \frac{F_{1}{ }^{2}}{2} h_{1} \frac{\xi_{1}{ }^{2}-1}{\xi_{1}{ }^{2}}+\frac{F_{2}{ }^{2}}{2} \frac{\rho_{2}}{\rho_{1}} h_{2} \frac{\xi_{2}{ }^{2}-1}{\xi_{2}{ }^{2}} \\
& +\left(H-H^{\prime}\right)+\frac{\Delta \rho}{\rho}\left(h_{2}-h_{2}{ }^{\prime}\right) \cdots \cdots
\end{aligned}
$$

From eq. (37) eq. (39) is transformed to

$$
\Delta E=\frac{F_{1}{ }^{2}}{2} h_{1} \frac{\xi_{1}{ }^{2}-1}{\xi_{1}{ }^{2}}+\frac{F_{2}{ }^{2}}{2} \frac{\rho_{2}}{\rho_{1}} h_{2} \frac{\xi_{2}{ }^{2}-1}{\xi_{2}{ }^{2}}
$$

If there is no discharge in the lower layer, we obtain an equation of $\Delta E$ in such a simple form.

$$
\Delta E=\frac{F_{1}^{2}}{2} h_{1} \frac{\xi_{1}^{2}-1}{\xi_{1}^{2}}
$$

For a stable internal jump to exist $\Delta E$ must have a positive value, so that $\xi_{1}$ must be larger than unity.

\subsection{Two Applications of the Internal Jump Theory}

I) From the industrial point of view there are some problems on the diffusion of industrial waste water into the $\mathrm{sea}^{6,7)}$. Usually we solve this problem by heat conduction type equation, ignoring the effect of density gradient. The diffusion coefficient is usually constant. The author has experimented on this problem in an idealized model the pure water flows from a channel into a pool of salt water; this is shown in Fig. 10 schematically.

Where there is no density difference between flowing water and stored water, Tollmien has solved the problem analytically on the assumption of mixing length theory. Fig. 9 shows the experimental results by the case of same densi$\mathrm{ty}^{7}{ }^{\text {) }}$. Where Froude number in the channel is within the range of $(0.1,0.5), \tan \theta$ seems

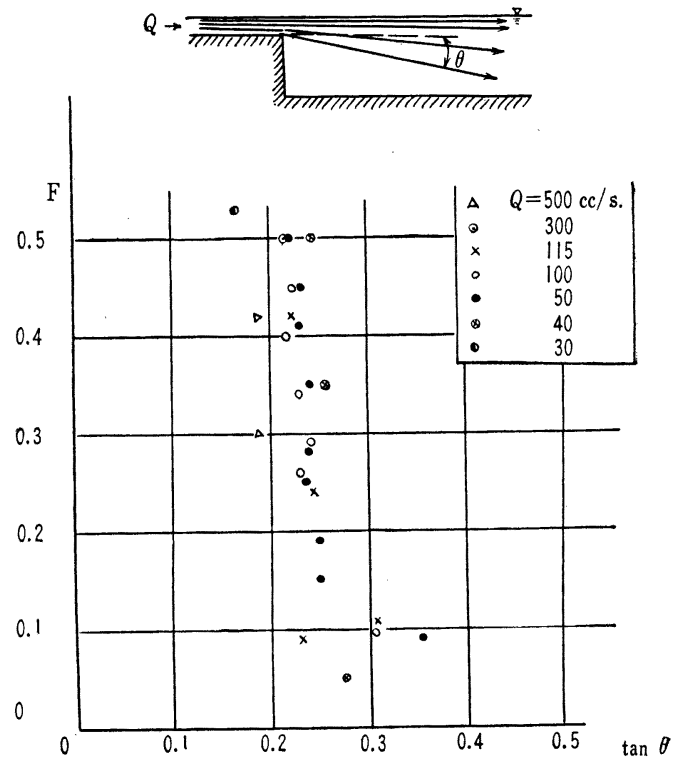

Fig. 9 Diffusion angle of two-dimensional jet (where there is no density difference).

constant, which Tollmien pointed out.

Where there is density difference, the phenomena differ from those mentioned above. Fig. 10 explains it. Pure water from the inlet keeps its initial depth for a while and suddenly changes it. As internal Froude numder decreases the depth change $\Delta h_{1}$ diminishes until zero. If we take this phenomenun as an internal jump, we may have the following analysis on it.

Using the fundamental equation of the internal jump, we may write the ratio of the stable depth $h_{1}^{\prime}$ and the initial depth $h_{1}$ as $\xi$.

$$
\xi_{1}=\frac{h_{1}{ }^{\prime}}{h_{1}}=\frac{1}{2}\left(-1+\sqrt{1+8 F_{i}{ }^{2}}\right)
$$

The condition that $\xi$ is larger than unity is

$$
F_{i}^{2}>1
$$

Fig. 11 shows the relation between experimental data and the theoretical curve. Were it not for 


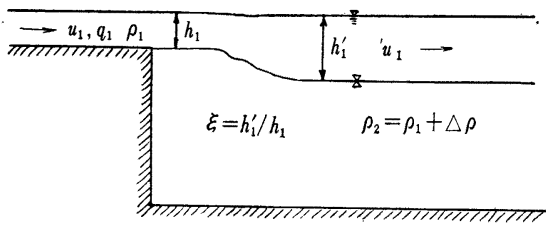

Fig. 10 Two-dimensional jet with density difference.

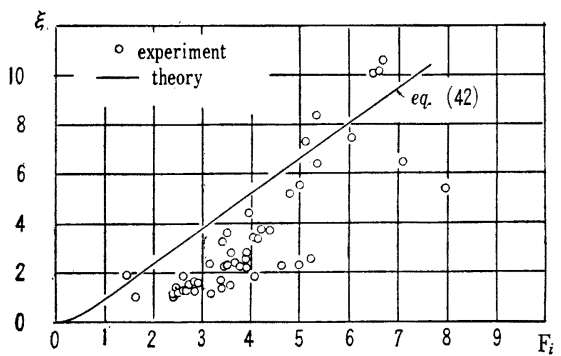

Fig. 11 Comparison between results of experiment and theory for normal internal jump.

any density change or mixing through the interface, experimental data should be lined on the theoretical curve of eq. (42).

II) In this paragraph the autheor shows some aspects on the structure of the mixing through the interface near the intake.

By selective withdrawal from the upper-layer there are two kinds of mixing structure through the interface. One is the mixing far from the intake; this is caused by breaking of internal waves $^{218)}$. The other is the mixing near the intake $^{9)}$; this is considered as a kind of internal jump. This time, however, $\Delta E<0$, since $\xi_{1}<1$ in eq. (41). Considering that $\Delta H$ can be expressed as $S \varepsilon\left(h_{1}-h_{1}{ }^{\prime}\right)$ before $h_{1}$ reaches conjugate value of $h_{1}{ }_{1}$, since $h^{\prime}{ }_{1}$ is the overflow depth of the intake weir, and keeps constant value under constant discharge, where $S$ is a parameter larger than unity. Fig. 12 is showing the situation by the weir type intake. So the condition that $\Delta E>0$ where $\xi_{1}<1$ is

$$
\Delta E=\varepsilon(\xi-1) h_{1}\left[\frac{F_{i}^{2}}{2} \frac{1+\xi}{\xi^{2}}-(S-1)\right]
$$

Fig. 13 shows the relation between $F_{i}$ and

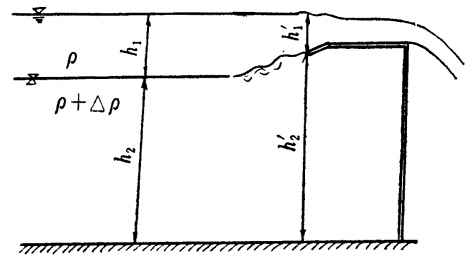

Fig. 12 Mixing near the intake. the mixing intensity $\rho_{o}-\rho_{e}$, where $\rho_{o}-\rho_{e}$ is difference of salt concentration between of water before the intake and of water after the intake. These are experimental data by the author and Mr. B. Yanagawa and two-layer system was of salt water and pure water; salt concentrition was measured by silver nitrate titration. From

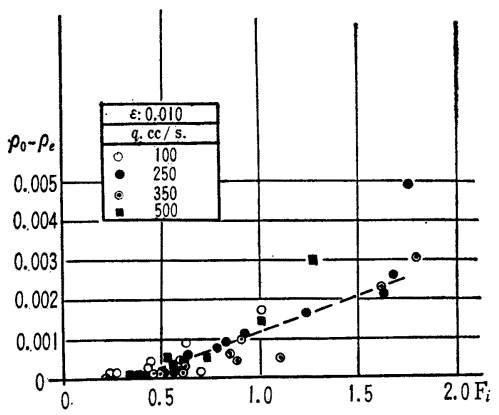

Fig. 13 Mixing intensity and internal Froud number $F_{i}$.

this result the mixing intensity is approximately linear and proportional to $F_{i}$.

Now that $F_{i}$ is

$$
F_{i}^{2}<2 \frac{\xi^{2}}{1+\xi}(S-1)
$$

$F_{i}$ is proportional to $q_{1}$, where $q_{1}$ is discharge of the upper layer per unit width. On the other hand the overflow depth of the weir $h_{1}$ is proportional to $q_{1}{ }^{2 / 3}$, so that from eq. (45) we obtain

$$
F_{i}=\frac{q_{1}}{g^{\prime 1 / 2}}\left(h_{1}\right)^{-2 / 3}
$$

where $c$ is the overflow confficient and is to be determined experimentally. The $c$-value of the used weir is $0.117(\mathrm{sec} / \mathrm{cm})$ and Fig. 14 shows

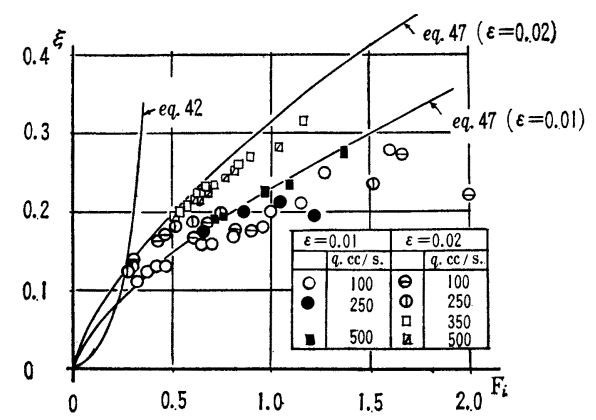

Fig. 14 Relation of and $F_{i}$ with some theoretical curves and Experimental results.

curves of $\xi$ and $F_{i}$ in case of $\frac{\Delta \rho}{\rho}=0.01$ and 0.02 , Data are lined up along the curve. As $F_{i}$ is getting larger, data are lined under the curve; 
this is perhaps caused by mixing in the channel.

On the other hand the theoretical curve calculated from eq. (36) is also shown in Fig. 14, and where $F_{i}$ is considerable large, $\xi$ calculated from eq. (36) is larger than that from eq. (46). We see from Fig. 13 and Fig. 14 that, where $s$ is larger than unity, mixing near the intake hardly occurs; this means energy change may seem one of the reasons of mixing near the intake. If it is so, we can easily calculate the critical internal Froude unmber from eq. (36) and eq. (46). This is a function with respect to $\frac{\Delta \rho}{\rho}$ and $c$.

$$
F_{i}=C^{-3 / 2} g^{-1 / 2} \xi^{3 / 2}
$$

For example let $c=0.117$ and $\frac{\Delta \rho}{\rho} g=10$, and we get $F_{i}{ }^{*}=0.202$.

The author believes we may have following conclusions.

1. The weir effective to selective-withdrawal must have large $c$-value.

2. If $\frac{\Delta \rho}{\rho}$ is getting larger, $F_{i}^{*}$ or discharge from the upper layer becomes large, so that the selective withdrawal from the upper layer gets on. (If we consider about mixing caused by breaking of internal waves, this seems more effective. Here we get a theoretical explanation by mixing near the intake.)

\section{REFERENCES}

1) Taylor, G.I. : Effect of Variation in Density on the Stability of Superposed Streams of Different Densities, Proc. of Roy. Soc. Aug. 1931.

2) Keulegan, G.H. : Interfacial Instability and Mixing in Stratified Flows, Jour. of Res. Vol. 43, 1949.

3) Schijf, J.B. \& Schönfeld, J.C. : Theoretical Consideration on the Motion of Salt and Fresh Water, Proc. of I.A.H.R. at Minesota, Sept. 1953.

4) Poggi, B.: Sul Moto Delle Correnti Stratificate, L'Energia Elettrica n. 3, 1959.

5) Yih Chia-Shun \& Guha, C.R. : Hydraulic Jump in a Fluid System of Two Layers, Tellus, n. 3, 1955.

6) Harleman, D.R.F. \& Ippen, A.T.: The Turbulent Diffusion and Convection of Saline Water in an Idealized Estuary, Comission of surface Water pp. 362-378.

7) Pai, S.I. : Two Dimensional Jet Mixing of Two Gases at Contant Temperature., Jour. of Appl. Mech. 22, No. 1, March 1955.

8) Craya, A. : Critical Regimes of Flow with Density Stratification., Tellus, Vol. 3, n. 1, 1951.

9) Huben, D.G. : Irrotational Motion of Two Fluid Strata towards a Line Sink, A.S.C.E. Eng. Mech. Div. Aug. 1960. 